TU-831

UT-HET-017

November, 2008

\title{
Synchrotron Radiation from the Galactic Center in Decaying Dark Matter Scenario
}

\author{
Koji Ishiwata $^{(a)}$, Shigeki Matsumoto ${ }^{(b)}$, Takeo Moroi ${ }^{(a)}$ \\ ${ }^{(a)}$ Department of Physics, Tohoku University, Sendai 980-8578, Japan \\ (b) Department of Physics, University of Toyama, Toyama 930-8555, Japan
}

\begin{abstract}
We discuss the synchrotron radiation flux from the Galactic center in unstable dark matter scenario. Motivated by the anomalous excess of the positron fraction recently reported by the PAMELA collaboration, we consider the case that the dark matter particle is unstable (and long-lived), and that energetic electron and positron are produced by the decay of dark matter. Then, the emitted $e^{ \pm}$becomes the source of the synchrotron radiation. We calculate the synchrotron radiation flux for models of decaying dark matter, which can explain the PAMELA positron excess. Taking the lifetime of the dark matter of $O\left(10^{26} \mathrm{sec}\right)$, which is the suggested value to explain the PAMELA anomaly, the synchrotron radiation flux is found to be $O(1 \mathrm{kJy} / \mathrm{str})$ or smaller, depending on the particle-physics and cosmological parameters.
\end{abstract}

\footnotetext{
*E-mail: ishiwata@tuhep.phys.tohoku.ac.jp

${ }^{\dagger}$ E-mail: smatsu@sci.u-toyama.ac.jp

${ }^{\ddagger}$ E-mail: moroi@tuhep.phys.tohoku.ac.jp
} 


\section{Introduction}

Nowadays there is no doubt about the existence of dark matter in our universe. However, nobody knows what dark matter is. The answer for the question will give a great impact on various fields of physics including astrophysics, cosmology and particle physics. For astrophysics and cosmology, dark matter is the dominant source of gravitation, and it governs the structure formation of our universe. For particle physics, we cannot find the candidate for dark matter in the standard model, so that dark matter is now regarded as the first evidence of new particles beyond the standard model.

Experimentally, many attempts have been made to understand the property of dark matter. Among them, the PAMELA collaboration has recently reported quite an interesting result; the anomalous excess of positrons with the energy of $20-100$ $\mathrm{GeV}$ in the cosmic ray [1]. The excess is hardly understood by the conventional mechanism [2, 3], namely the secondary production of positrons due to the collision between primary protons and interstellar medium in our Galaxy.

Theoretically, a lot of scenarios for dark matter have been proposed to explain the anomalous excess of positrons. These scenarios are categorized into two types; positron productions from the annihilation of dark matter [4, 5, 6, 17, 8, 9, 10, 11] and from the decay of dark matter [12, 13, 14, 15, 16, 17]. In most of the scenarios in the former case, people have faced to the difficulty to explain the excess; a huge enhancement factor, called boost factor, to the production rate of positrons is needed, where the factor is from the inhomogeneity of the dark matter distribution in the vicinity of the solar system. \#1 On the contrary, in the latter case, the positron flux to explain the excess can be naturally obtained with the lifetime of dark matter much longer than the age of the universe. One might worry about the fine-tuning problem to have a long lifetime of dark matter, however, there are some scenarios to realize such a long lifetime, as we discuss in the following.

Synchrotron radiation from the annihilation or decay of dark matter may give constrains directly to scenarios aiming to the explanation of the PAMELA positron excess. Since dark matter annihilates or decays into energetic positron (as well as electron) under the circumstance of magnetic fields in our galaxy, synchrotron

\footnotetext{
${ }^{\# 1}$ There also be some exceptions, for instance, a huge annihilation cross section can be obtained if the Sommerfelt enhancement to the cross section is considered [18, 19, 20. A large boost factor is not mandatory in those scenarios
} 
radiation is inevitably induced. Importantly, the WMAP collaboration has observed the radiation in the whole sky, so that the observation gives constraints on the scenarios of the electron and positron production due to the annihilation or decay of dark matter in the Galactic halo. The synchrotron radiation flux for the case of dark matter annihilation has been considered in past works [21, 22]. The purpose of this article is to study the synchrotron radiation from the decay of dark matter, and discuss implication to the scenarios of decaying dark matter which account for the PAMELA result.

The organization of this paper is as follows. In the next section, we explain the cosmological scenario we consider. The formalism to calculate the synchrotron flux from the decay of dark matter is given in Section 3. Compared to the previous works, several improvements are realized. Then, we numerically calculate the flux for several dark matter models; the results are shown in Section 4 . The summary of our study is given in Section 5 .

\section{The Scenario}

In this section, we summarize the cosmological scenario we consider. As we mentioned in the introduction, taking the anomalous excess of the positron fraction measured by the PAMELA [1] seriously, we consider unstable dark matter scenario. Indeed, if the decay of dark matter produces energetic positron, it becomes the source of the cosmic ray positron, and may explain the excess of the positron fraction.

In the following, we consider the case that the dark matter particle (which we denote $X$ ) is unstable, and that its decay produces energetic positron (as well as electron). We also assume that the energetic positron produced by the decay is the origin of the excess of the positron fraction observed by the PAMELA. So far, many possibilities of such an unstable dark matter has been discussed [12, 13, 14, 15, 16, 17]. The energy spectrum of the produced $e^{ \pm}$depends on the model; in some cases, only the monochromatic electron and positron are produced by the decay and, in other cases, the energy distribution of the $e^{ \pm}$is non-monochromatic.

The positron flux from the dark matter decay is inversely proportional to the lifetime of dark matter $\tau_{\mathrm{DM}}$. The best-fit value of $\tau_{\mathrm{DM}}$ to explain the PAMELA anomaly depends on the energy spectrum of the positron from the dark matter decay. For example, if the dark matter mostly decays as $X \rightarrow e^{+} e^{-}$, the PAMELA 
anomaly is well explained when [13]

$$
\tau_{\mathrm{DM}} \simeq 2.2 \times 10^{27} \mathrm{sec} \times\left(\frac{m_{\mathrm{DM}}}{100 \mathrm{GeV}}\right)^{-1} .
$$

For example, if the sneutrino lightest superparticle (LSP) in the supersymmetric model is dark matter and also if it decays via an $R$-parity breaking superpotential interaction, this is the case $\# 2$ On the contrary, if dark matter is the unstable gravitino which dominantly decays into $l_{i}^{ \pm} W^{\mp}$ final state (with $l_{i}^{ \pm}$being charged lepton in $i$-th generation) [25, 26], $e^{ \pm}$is also produced by the decay of $W^{ \pm}$. In such a case, the best-fit lifetime becomes

$$
\tau_{\mathrm{DM}} \simeq 8.5 \times 10^{26} \mathrm{sec} \times\left(\frac{m_{\mathrm{DM}}}{100 \mathrm{GeV}}\right)^{-1}
$$

In any case, in order to explain the PAMELA anomaly, the lifetime of $O\left(10^{26-27} \mathrm{sec}\right)$ is needed (when $m_{\mathrm{DM}} \sim O(100 \mathrm{GeV})$ ). In the following, we concentrate on the case that $\tau_{\mathrm{DM}}$ is of this order. In addition, as we will discuss, taking these values of the lifetime and $m_{\mathrm{DM}} \sim 1 \mathrm{TeV}$, the $\left(e^{-}+e^{+}\right)$flux becomes consistent with those reported by the ATIC [27] and the PPB-BETS [28] experiments, which have observed excess of the flux at the energy of a few hundred GeV.

Once the electron and positron are emitted by the decay of dark matter in our Galaxy, they cause the synchrotron radiation and the emitted radiation may be observed. In the following, we calculate the flux of the synchrotron radiation from the central region of our Galaxy.

\section{Synchrotron Radiation Flux}

In this section, we formulate the calculation of the radio flux from synchrotron radiation generated by high energy electron (and positron). First, we discuss the propagation of the electron and positron produced by the decay in the Galaxy. Then, we summarize the formula for the calculation of the synchrotron radiation.

\subsection{Propagation model for the electron and positron}

The synchrotron radiation flux depends on the energy spectra of the electron and positron (at each point of the Galaxy) produced by the dark matter decay. We first discuss the propagation of the electron and positron in the Galaxy to understand

\footnotetext{
\#2Even if the sneutrino is dark matter, the direct-detection constraint can be avoided. See [23, 24].
} 


\begin{tabular}{lcccc}
\hline \hline Model & $\delta$ & $K_{0}\left(\mathrm{kpc}^{2} / \mathrm{Myr}\right)$ & $L(\mathrm{kpc})$ & $R(\mathrm{kpc})$ \\
\hline M1 & 0.46 & 0.0765 & 15 & 20 \\
MED & 0.70 & 0.0112 & 4 & 20 \\
M2 & 0.55 & 0.0060 & 1 & 20 \\
\hline \hline
\end{tabular}

Table 1: Parameters for the propagation models of electron, which will be used in our numerical calculations.

how the spectra are determined. For the electron and positron from the decay of dark matter, their spectra are expected to be the same, so we consider the electron spectrum.

When the energetic electron is emitted, its trajectory is twisted in the so-called diffusion zone where the magnetic field is non-negligible. Because the magnetic field is entangled, the motion of the electron is expected to be well approximated by a random walk. Then, the propagation of the electron is described by the following differential equation:

$$
K(E) \nabla^{2} f_{e}(E, \vec{x})+\frac{\partial}{\partial E}\left[b(E, \vec{x}) f_{e}(E, \vec{x})\right]+Q(E, \vec{x})=0
$$

where $f_{e}$ is the electron spectrum (i.e., number density of the electron per unit energy), $K(E)$ is the diffusion coefficient, $b(E, \vec{x})$ is the energy loss rate, and $Q(E, \vec{x})$ is the electron source term.

Diffusion of injected electron is caused by the entangled magnetic field in the Galaxy. The function $K(E)$ can be determined so that the cosmic-ray Boron to Carbon ratio and sub-Fe to Fe ratio are reproduced. In our analysis, we adopt the propagation model given in [29], where the shape of the diffusion zone is approximated by a cylinder (with the radius $R$ and the half-height $L$ ) and the function $K(E)$ is parametrized as

$$
K(E)=K_{0}\left(\frac{E}{1 \mathrm{GeV}}\right)^{\delta} .
$$

Parameters of three representative models, MED, M1, and M2 models, are shown in Table 1. The MED model is the best-fit to the Boron-to-Carbon ratio analysis, while the maximal and minimal positron fractions for $E \gtrsim 10 \mathrm{GeV}$ are expected to be estimated with M1 and M2 models, respectively.

The energy loss of electron is via synchrotron radiation under the magnetic field and inverse Compton scatterings with cosmic microwave background (CMB) and 
infrared gamma ray from stars. Then, $b(E, \vec{x})$ is given by the sum of the synchrotron energy loss rate $P_{\text {synch }}$ and the inverse Compton energy loss rate $P_{\mathrm{IC}}$ :

$$
b(E, \vec{x})=P_{\text {synch }}+P_{\mathrm{IC}} \equiv P_{\text {synch }}\left[1+r_{\mathrm{IC} / \text { synch }}(\vec{x})\right] .
$$

For simplicity, we neglect the position dependence of the magnetic flux density $B$ in the diffusion zone; then, $P_{\text {synch }}$ becomes uniform in the diffusion zone. Since the magnetic flux in the Galaxy is not well understood, we take several values of $B$ to see how the synchrotron radiation flux depends on the magnetic field in the following numerical analysis. The synchrotron energy loss rate $P_{\text {synch }}$ is given by [30, 31]

$$
P_{\text {synch }}=\frac{e^{4} E^{2} B^{2}}{6 \pi \epsilon_{0} m_{e}^{4} c^{5}} \simeq 3.4 \times 10^{-17} \mathrm{GeVsec}^{-1} \times\left(\frac{E}{1 \mathrm{GeV}}\right)^{2}\left(\frac{B}{3 \mu \mathrm{G}}\right)^{2},
$$

where $e$ is the electron electric charge, $\epsilon_{0}$ is the permittivity of free space, $m_{e}$ is the electron mass, and $c$ is the speed of light. In addition, $r_{\mathrm{IC} / \text { synch }}$ is expressed as

$$
r_{\mathrm{IC} / \mathrm{synch}}(\vec{x})=\frac{2}{3} \frac{U_{\mathrm{rad}}(\vec{x})}{\left(B^{2} / 2 \mu_{0}\right)} \simeq 2.7 \times\left(\frac{U_{\mathrm{rad}}}{0.9 \mathrm{eVcm}^{-3}}\right)\left(\frac{B}{3 \mu \mathrm{G}}\right)^{-2},
$$

where $U_{\text {rad }}$ is the radiation density, and $\mu_{0}$ is the permeability of free space. In order to take into account the position dependence of $U_{\text {rad }}$, we use the results given in [32] where the radiation density on the Galactic disc (i.e., $U_{\text {rad }}\left(r_{\|}, z=0\right)$ ) and that on the surface of $r_{\|}=4 \mathrm{kpc}$ (i.e., $U_{\text {rad }}\left(r_{\|}=4 \mathrm{kpc}, z\right)$ ) are given, where $z$ and $r_{\|}$are the height and the radial distance in the cylindrical polar coordinate. We assume the following formula for the radiation density (unless otherwise mentioned):

$$
U_{\mathrm{rad}}\left(r_{\|}, z\right)=\frac{U_{\star}(4 \mathrm{kpc}, z)}{U_{\star}(4 \mathrm{kpc}, 0)} U_{\star}\left(r_{\|}, 0\right)+U_{\mathrm{CMB}},
$$

with $U_{\mathrm{CMB}} \simeq 0.3 \mathrm{eVcm}^{-3}$ being the $\mathrm{CMB}$ contribution to the radiation density. In addition, $U_{\star} \equiv U_{\text {rad }}-U_{\mathrm{CMB}}$ is the radiation density from stars, which is determined from the results given in [32]. Then, one can see that $U_{\text {rad }}$ varies as $0.3-10 \mathrm{eVcm}^{-3}$ from the rim to the center of the Galaxy. Notice that, in many previous works, $U_{\text {rad }}$ is approximated to be a constant. However, the position dependence of $U_{\text {rad }}$ gives a significant effect on the resultant synchrotron radiation flux because the inverse Compton scattering may become the dominant energy-loss process.

The electron source term is given by electron injection rate and dark matter distribution in the Milky Way Galaxy halo as

$$
Q(E, \vec{x})=\frac{1}{\tau_{\mathrm{DM}}} \frac{\rho_{\mathrm{DM}}(\vec{x})}{m_{\mathrm{DM}}} \frac{d N_{e}}{d E}
$$


where $\rho_{\mathrm{DM}}$ is energy density of dark matter and $d N_{e} / d E$ is energy distribution of electron from the decay of single $X$. For the dark matter distribution $\rho_{\mathrm{DM}}$, we use the isothermal halo density profile:

$$
\rho_{\text {halo }}(r)=\rho_{\odot} \frac{r_{\text {core }}^{2}+r_{\odot}^{2}}{r_{\text {core }}^{2}+r^{2}},
$$

where $\rho_{\odot} \simeq 0.43 \mathrm{GeV} / \mathrm{cm}^{3}$ is the local halo density, $r_{\text {core }} \simeq 2.8 \mathrm{kpc}$ is the core radius, $r_{\odot} \simeq 8.5 \mathrm{kpc}$ is the distance between the Galactic center and the solar system, and $r$ is the distance from the Galactic center.

Before solving the diffusion equation (3.1), it is instructive to estimate the typical propagation length of the electron per time scale of the energy loss, which we denote $\langle l(E)\rangle$. For the electron with energy $E$, the time scale of the energy loss is $\sim E / b(E)$, and hence we obtain $\langle l(E)\rangle \sim \sqrt{K E / b}$. For the propagation model given in Table 1. $\langle l(E)\rangle$ becomes a few kpc for $E \sim 1 \mathrm{GeV}$ (where we have used the energy loss rate given in (3.2)), and it becomes shorter as the energy increases. For such a scale, the change of the dark matter density is not significant (except for the central region of the Galaxy). In particular, if the effect of the propagation is negligible, we can omit the term proportional to $K(E)$ in the diffusion equation and, in such a case, we obtain the following approximated formula for the electron spectrum:

$$
f_{e}^{(\text {local })}(E, \vec{x})=\frac{1}{\tau_{\mathrm{DM}}} \frac{\rho_{\mathrm{DM}}(\vec{x})}{m_{\mathrm{DM}}} \frac{Y_{e}(>E)}{b(E, \vec{x})},
$$

where

$$
Y_{e}(>E) \equiv \int_{E}^{\infty} d E^{\prime} \frac{d N_{e}}{d E^{\prime}}
$$

Thus, in such a case, the energy spectrum at the point $\vec{x}$ is almost determined by the local dark matter density at the same point.

In Fig. 1, we plot the electron flux $\Phi_{e} \equiv \frac{c}{4 \pi} f_{e}$ at the solar system (i.e., $r=r_{\odot}$ and $z=0)$. Here, we consider the case that $\operatorname{Br}\left(X \rightarrow e^{+} e^{-}\right)=1$, taking $m_{\mathrm{DM}}=200 \mathrm{GeV}$ and $600 \mathrm{GeV}, \tau_{\mathrm{DM}}=5 \times 10^{26} \mathrm{sec}$, and $B=3 \mu \mathrm{G}$; then, the energy distribution in Eq. (3.6) is

$$
\frac{d N_{e}}{d E}=\delta\left(E-m_{\mathrm{DM}} / 2\right)
$$

In addition, in depicting Fig. 1, we use $U_{\text {rad }} \simeq 0.9 \mathrm{eV} \mathrm{cm}^{-3}$ for simplicity. One can see that the flux $f_{e}^{(\text {local })}$ well agrees with the predictions of the MED and M1 models for $E \gtrsim 10 \mathrm{GeV}$, which confirms our earlier discussion. On the other hand, one 

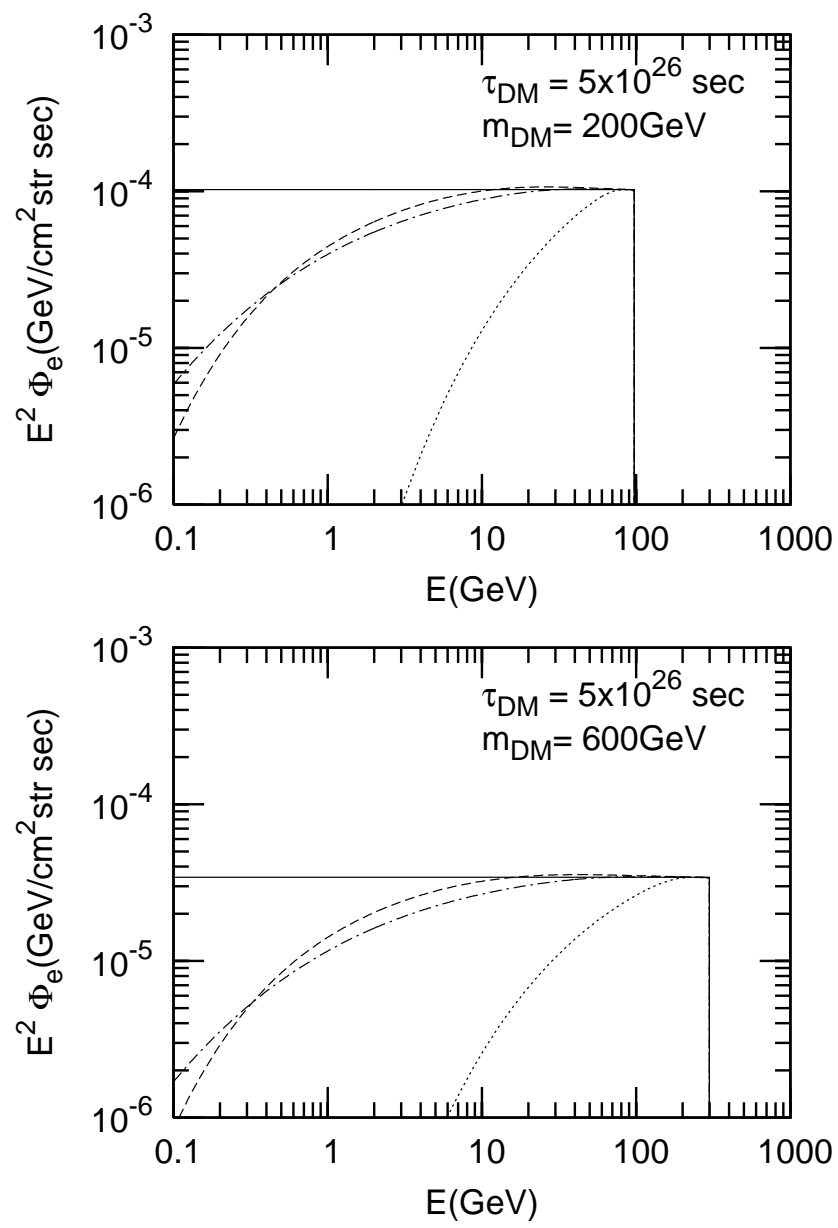

Figure 1: Electron fluxes in MED (dashed), M1 (dot-dashed), and M2 (dotted) models, and that from $f_{e}^{\text {(local) }}$ (solid), for the case that $\operatorname{Br}\left(X \rightarrow e^{+} e^{-}\right)=1$. Here, we take $m_{\mathrm{DM}}=200 \mathrm{GeV}$ (top) and $600 \mathrm{GeV}$ (bottom), $\tau_{\mathrm{DM}}=5 \times 10^{26} \mathrm{sec}, B=3 \mu \mathrm{G}$, and $U_{\mathrm{rad}}=0.9 \mathrm{eV} \mathrm{cm}^{-3}$.

can observe a significant difference between $f_{e}^{(\text {local })}$ and the flux in the M2 model. This is due to the smallness of half-height of diffusion zone in the M2 model, which is $1 \mathrm{kpc}$; with such a small half-height, the electron can escape from the diffusion zone. Given the fact that the MED model is the best fit to the cosmic-ray Boron to Carbon ratio analysis, we adopt the MED model as our benchmark model of the electron propagation. 


\subsection{Synchrotron radiation: formalism}

Synchrotron radiation energy density per unit time and unit frequency is expressed as

$$
L_{\nu}(\vec{x})=\int d E \mathcal{P}(\nu, E) f_{e}(E, \vec{x})
$$

Here, $\mathcal{P}(\nu, E)$ is synchrotron radiation energy per unit time and unit frequency from single electron with energy $E$, which is given by [30, 31]

$$
\mathcal{P}(\nu, E)=\frac{1}{4 \pi \epsilon_{0}} \frac{\sqrt{3} e^{3} B}{m_{e} c} F\left(\nu / \nu_{c}\right),
$$

where $\nu_{c}$ is critical frequency defined as

$$
\nu_{c} \equiv \frac{3 e E^{2} B}{4 \pi m_{e}^{3} c^{4}}
$$

and

$$
F(x) \equiv x \int_{x}^{\infty} d y K_{5 / 3}(y)
$$

with $K_{z}$ being the modified Bessel function of $z$-th order \#3 The function $F\left(\nu / \nu_{c}\right)$ has a peak at $\nu \simeq 0.29 \nu_{c}$. Adopting the Galactic magnetic flux density of $B \sim 3 \mu \mathrm{G}$, we can see that the synchrotron radiation in the observed frequency band of the WMAP (i.e, $22-93 \mathrm{GHz}$ ) is from the electron with the energy of $E \sim 10-100 \mathrm{GeV} \# 4$ For the electron in such an energy range, as we have seen, the electron spectrum with the MED model, which is our benchmark model, is well approximated by $f_{e}^{(\text {local })}$ given in Eq. (3.8). Thus, we use $f_{e}^{\text {(local) }}$ in our numerical calculation.

In order to calculate the observed radiation energy flux, we integrate $L_{\nu}(\vec{x})$ along the line of sight (l.o.s.), whose direction is parametrized by the parameters $\theta$ and

\#3 This formula, as well as energy loss rate given in Eq. (3.3), is for the case that the velocity of electron is perpendicular to the magnetic field. Of course, such situation is not always satisfied in the Galaxy. Therefore, considering the fact that the injected electron is isotropic and the magnetic field is entangled, we take the $B$ in the formula as mean magnetic flux density which the electron feels effectively.

\#4 In the previous works [33, 34, 35, 36, 37], $F(x)$ is approximated as

$$
F(x) \simeq \frac{8 \pi}{9 \sqrt{3}} \delta(x-0.29) .
$$

However, we found that such an approximation is not good in particular when $Y_{e}(>E)$ is suppressed for $E \gtrsim \sqrt{4 \pi m_{e}^{3} c^{4} \nu / 3 e B}$. 
$\phi$, where $\theta$ is the angle between the direction to the Galactic center and that of the line of sight, and $\phi$ is the rotating angle around the direction to the Galactic center. (The Galactic plane corresponds to $\phi=0$ and $\pi$.) Then, the synchrotron radiation flux is given by

$$
J_{\nu}(\theta, \phi)=\frac{1}{4 \pi} \int_{\text {l.o.s. }} d \vec{l} L_{\nu}(\vec{l})
$$

We note here that we do not consider the synchrotron radiation from the very central region of the Galaxy and that the effect of the self absorption is unimportant [36]. Using Eqs. (3.8) and (3.12), we obtain the energy flux from synchrotron radiation as

$$
J_{\nu}(\theta, \phi)=\frac{9 \sqrt{3}}{32 \pi^{2}} \frac{1}{m_{\mathrm{DM}} \tau_{\mathrm{DM}}} \int_{\text {l.o.s. }} d \vec{l} \frac{\rho_{\mathrm{DM}}(\vec{l})}{1+r_{\mathrm{IC} / \text { synch }}(\vec{x})} \int d E \frac{Y_{e}(>E)}{\nu_{c}} F\left(\nu / \nu_{c}\right) .
$$

Notice that, adopting the approximation of the constant magnetic flux in the Galaxy, the line of sight and energy integrals factorize.

\section{Numerical Results}

Now, we show the synchrotron radiation flux. We numerically evaluate the flux given in Eq. (3.16) for several dark matter models. In the following, we show the results for $\phi=\frac{\pi}{2}$, i.e., $J_{\nu}(\theta, \pi / 2)$. The frequency of the radiation is taken to be some of the WMAP frequency bands: $\nu=22,33$, and $61 \mathrm{GHz}$.

\subsection{Leptonically decaying dark matter}

Let us start with the simplest case where the dark matter particle dominantly decays as $X \rightarrow e^{+} e^{-}$. This is the case if, for example, the sneutrino field in supersymmetric model is the LSP, and also if the $\hat{L}_{i} \hat{L}_{1} \hat{E}_{1}$-type $R$-parity violating superpotential exists (where $\hat{L}_{i}$ and $\hat{E}_{i}$ are superfields for $S U(2)_{L}$ doublet- and singlet-lepton superfields, respectively) [13]. In this case, the energy distribution in Eq. (3.6) is given by Eq. (3.10). As we have mentioned, with such a flux, the PAMELA anomaly can be well explained if the lifetime of dark matter is properly chosen. (See Eq. (2.1).) In addition, when $m_{\mathrm{DM}}$ is larger than $\sim 100 \mathrm{GeV}$, the dark matter contribution to the electron and positron fluxes for the energy range of $100 \mathrm{GeV} \lesssim E \leq \frac{1}{2} m_{\mathrm{DM}}$ are approximately given by

$$
E^{2} \Phi_{e^{ \pm}} \simeq 4.5 \times 10^{-5} \mathrm{GeV} / \mathrm{cm}^{2} \sec \operatorname{str} \times\left(\frac{\tau_{\mathrm{DM}}}{2.2 \times 10^{26} \mathrm{sec}}\right)^{-1}\left(\frac{m_{\mathrm{DM}}}{1 \mathrm{TeV}}\right)^{-1}
$$




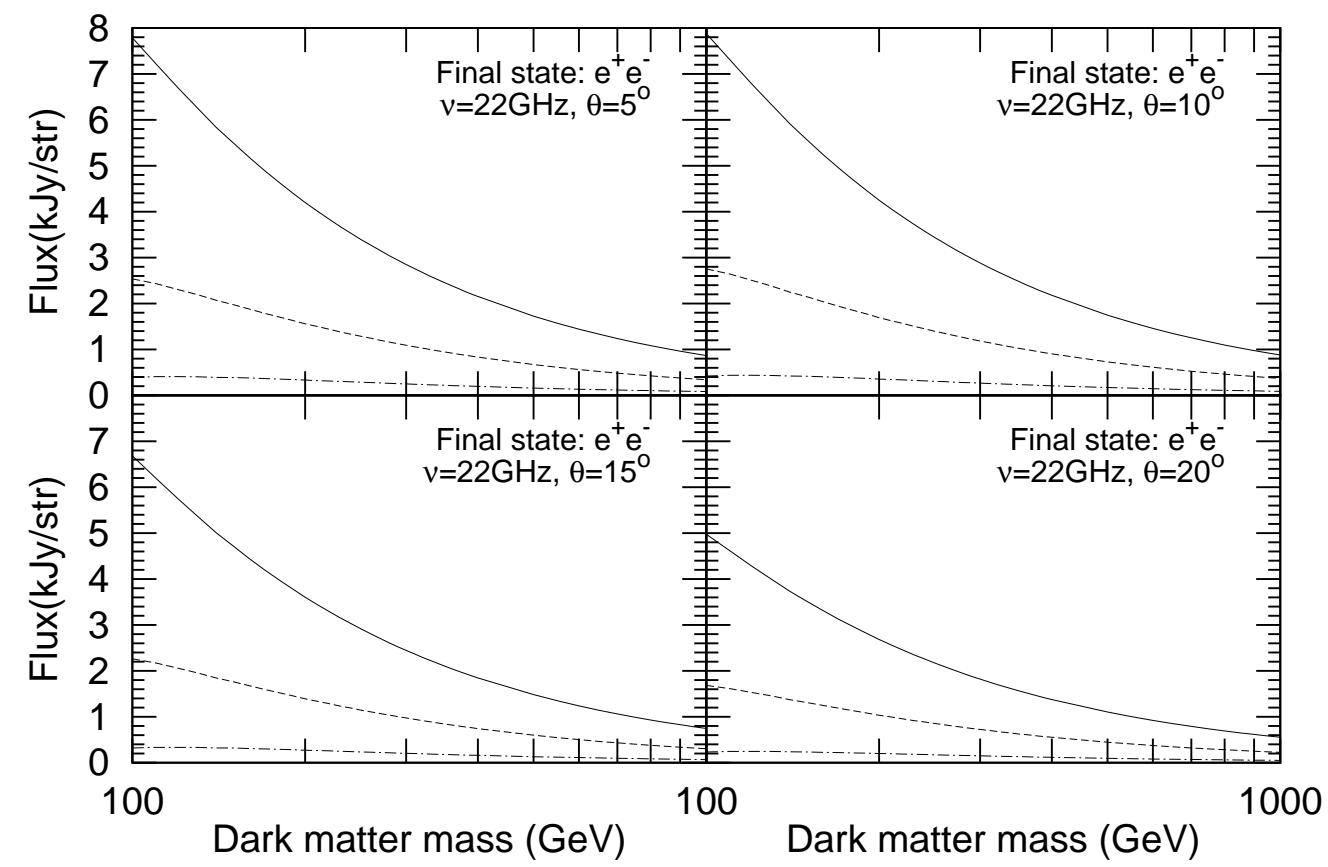

Figure 2: Synchrotron radiation fluxes at $\nu=22 \mathrm{GHz}$ as functions of dark matter mass for angle $\theta=5^{\circ}, 10^{\circ}, 15^{\circ}$, and $20^{\circ}$. Here, the dark matter is assumed to decay only into $e^{+} e^{-}$pair. Here, we take $\tau_{3 / 2}=5 \times 10^{26} \mathrm{sec}$, and show the cases of $B=1$, $3,10 \mu \mathrm{G}$ (from the bottom to the top) for each figure.

where the MED model of the propagation is used here. (The flux for $E \geq \frac{1}{2} m_{\mathrm{DM}}$ vanishes.) Then, if $m_{\mathrm{DM}} \sim 1 \mathrm{TeV}$, a bump at $E \sim 500 \mathrm{GeV}$ shows up in the $\left(e^{-}+e^{+}\right)$ flux, taking the best-fit lifetime to explain the PAMELA anomaly. The behavior is consistent with the excesses of the $\left(e^{-}+e^{+}\right)$flux observed by the ATIC [27] and the PPB-BETS [28] experiments.

In Fig. 2, we plot the fluxes for $\nu=22 \mathrm{GHz}$ as functions of $m_{\mathrm{DM}}$, for $\tau_{\mathrm{DM}}=$ $5 \times 10^{26}$ sec, which is of the same order of the lifetime suggested from the PAMELA anomaly $\# 5$ The angle $\theta$ is taken to be $5^{\circ}, 10^{\circ}, 15^{\circ}$, and $20^{\circ}$ (which give the minimum distance from the Galactic center to the line of sight of $0.7,1.5,2.3$, and $3.1 \mathrm{kpc}$, respectively), and $B=1,3$, and $10 \mu \mathrm{G}$. We also show the fluxes for $\nu=33$ and $61 \mathrm{GHz}$ in Figs. 3 and 4 , respectively.

As one can see, the synchrotron radiation flux from the decay is $O(1 \mathrm{kJy} / \mathrm{str})$ or smaller in wide parameter region $m_{\mathrm{DM}} \sim 100 \mathrm{GeV}-1 \mathrm{TeV}$. The flux $J_{\nu}$ decreases

\footnotetext{
\#5 The synchrotron radiation flux is inversely proportional to $\tau_{\mathrm{DM}}$ (see Eq.(3.16) ). In order to obtain the flux for different value of $\tau_{\mathrm{DM}}$, one can simply rescale the flux given in the figure.
} 


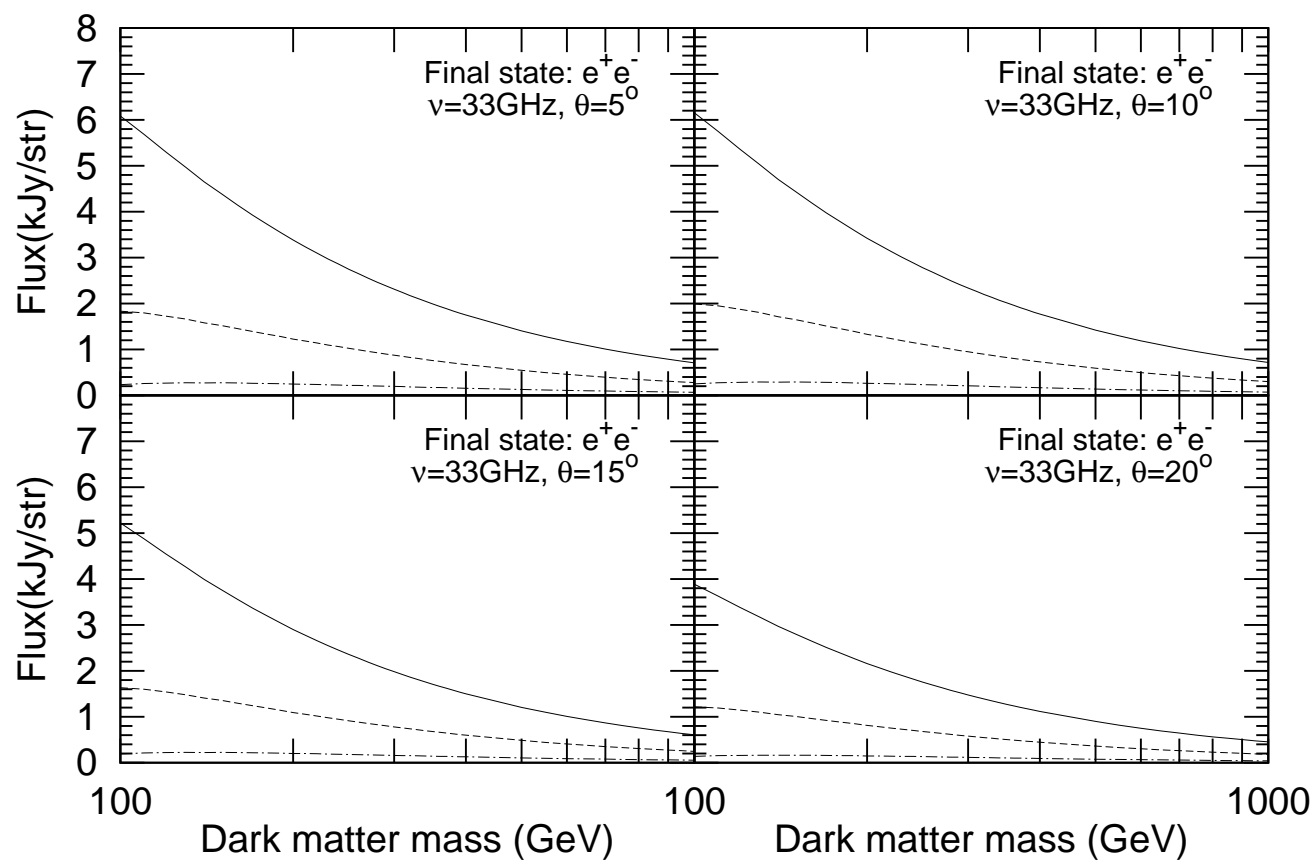

Figure 3: Same as Fig. 2, except for $\nu=33 \mathrm{GHz}$.

as the frequency increases. In addition, $J_{\nu}$ decreases as $B$ decreases; this is because, as the magnetic field becomes weaker, the $e^{ \pm} \mathrm{s}$ lose more energy by inverse Compton process, resulting in a suppression of the synchrotron radiation flux.

Radiation flux from Galactic center region has been observed by the WMAP for frequency bands of 22, 33, 41, 61, and $93 \mathrm{GHz}$ [38, 39]. Since then, intensive analysis has been performed to understand the origins of the radiation flux. (For recent studies, see [38, 21, 22, 39].) Most of the radiation flux is expected to be from astrophysical origins, such as thermal dust, spinning dust, ionized gas, and synchrotron radiation, which have been studied by the use of other survey data [40, 41, 42]. With the three-year data, the WMAP collaboration claimed that the flux intensity can be explained by the known astrophysical origins [43]. On the contrary, Refs. [21, 22, 38] also studied the WMAP three-year data, and claimed that there exists a remnant flux from unknown origin which might be non-astrophysical; the remnant flux is called the "WMAP Haze" \#6 However, no clear indication of the

\footnotetext{
${ }^{\# 6}$ In [21, 22, as a possible explanation for the WMAP Haze, annihilation of dark matter is proposed. If energetic charged particles are emitted via the dark matter annihilation, they become another source of the synchrotron radiation. According to the study, the synchrotron radiation flux turns out to be comparable to the WMAP Haze with the annihilation cross section $\langle\sigma v\rangle \sim$ $O\left(10^{-26} \mathrm{~cm}^{3} \mathrm{sec}^{-1}\right)$, which is consistent magnitude to explain dark matter abundance.
} 


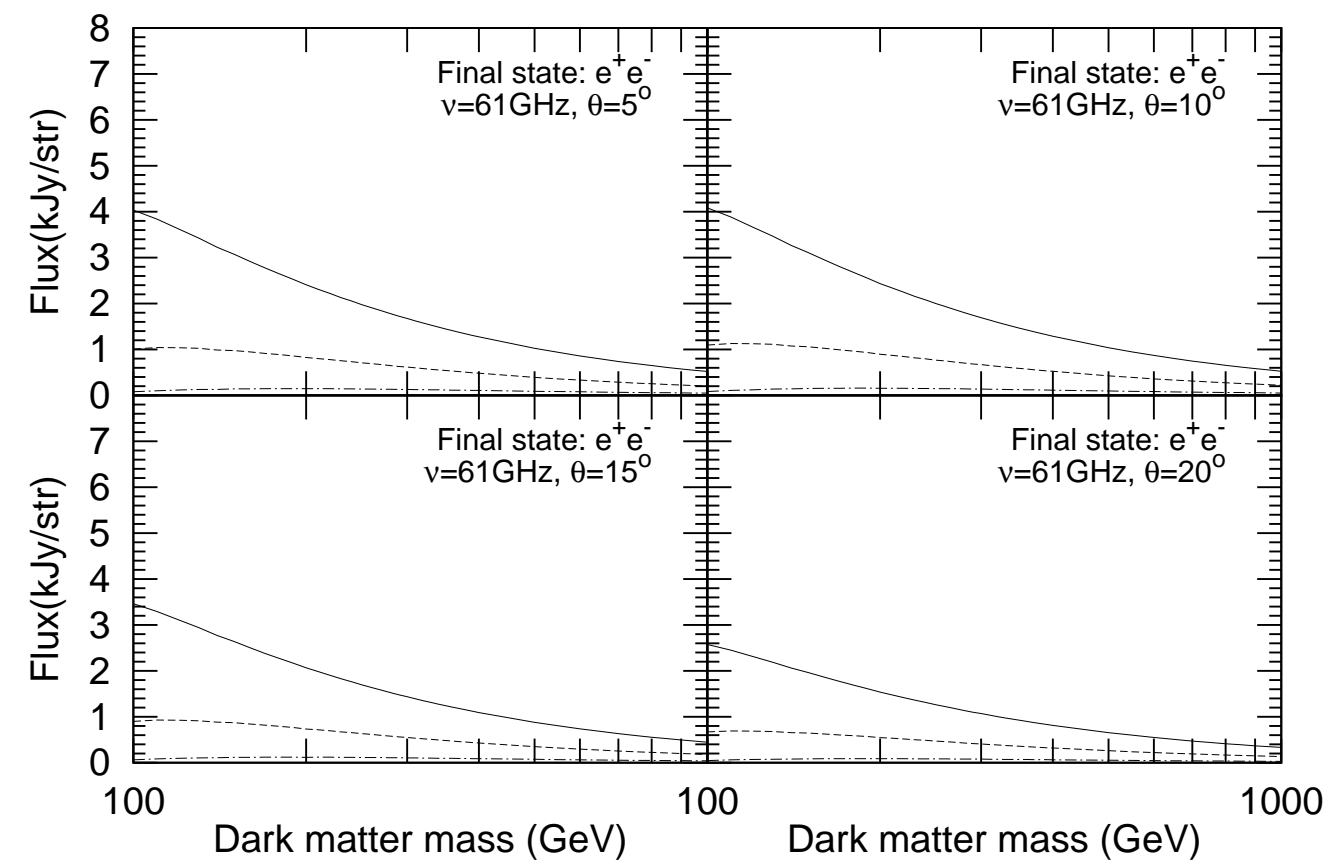

Figure 4: Same as Fig. 2, except for $\nu=61 \mathrm{GHz}$.

WMAP Haze from unknown source was reported by the WMAP collaboration after five-year data [39].

The existence of the WMAP Haze seems still controversial, and the detailed studies of the WMAP Haze using the data is beyond the scope of our study. Here, we adopt the flux of the WMAP Haze suggested in [21, 22] as a reference value. Our numerical calculation shows that the predicted flux, which is $O(1 \mathrm{kJy} / \mathrm{str})$, is comparable to the flux of the WMAP Haze given in [21, 22]. As we mentioned, since the the existence of the exotic radiation flux of this size is controversial, it is difficult to confirm or exclude the present scenario without better understandings of the sources of Galactic foreground emission.

\subsection{Gravitino dark matter}

Next, let us consider the case that the gravitino (which we denote $\psi_{\mu}$ ) is the LSP and is dark matter. Even if the gravitino is the LSP, it becomes unstable if the $R$-parity is violated. In particular, if the effect of the $R$-parity violation is small enough, the lifetime of the gravitino becomes longer than the present age of the universe and it is a viable candidate for dark matter [44]. Production of the gravitino may be due to the thermal scattering [45], the decay of the lightest superparticle in the minimal 
supersymmetric standard model sector [46, 47, 48, 49, 50], and the decay of the inflaton field [51]. We do not specify any particular scenario, but just assume that the density parameter of the gravitino has somehow become consistent with the dark matter density.

In this study, we consider the following $R$-parity violating operators:

$$
\mathcal{L}_{\mathrm{RPV}}=B_{i} \tilde{L}_{i} H_{u}+m_{\tilde{L}_{i} H_{d}}^{2} \tilde{L}_{i} H_{d}^{*}+\text { h.c. }
$$

where $\tilde{L}_{i}$ is left-handed slepton doublet in $i$-th generation, while $H_{u}$ and $H_{d}$ are upand down-type Higgs boson doublets, respectively. (We work in the basis where the bi-linear $R$-parity violating superpotential vanishes, which is realized by an appropriate redefinition of the Higgs and lepton-doublet chiral superfields.) With the abovementioned $R$-parity violating operators, the anomalous excesses of the positron [52, 1] and $\gamma$-ray [53] in the cosmic ray can be simultaneously explained when the parameters $B_{i}$ and $m_{\tilde{L}_{i} H_{d}}^{2}$ are properly chosen \#7

The flavor of the final-state lepton depends on the sizes of the $R$-parity violating coupling constants. In this analysis, for simplicity, we assume that the gravitino decays only into leptons in one of the three generations. Then, the gravitino decays as $\psi_{\mu} \rightarrow l_{f}^{-} W^{+}, \nu_{f} Z, \nu_{f} h$, and $\nu_{f} \gamma$ (where $f=1$, 2 , or 3 specifies the generation of the final-state lepton). In particular, the process $\psi_{\mu} \rightarrow l_{f}^{ \pm} W^{\mp}$ has the largest branching ratio (when the process is kinematically allowed). Notably, from this process, the charged lepton $l_{f}^{ \pm}$is directly produced, which results in an energetic electron or positron. If $l_{f}^{ \pm}=e^{ \pm}$, such an $e^{ \pm}$has energy of $\left(m_{3 / 2}^{2}-m_{W}^{2}\right) / 2 m_{3 / 2}$, where $m_{3 / 2}$ is the gravitino mass. In addition, the decays of the weak- and Higgs-bosons also produce $e^{ \pm}$. As we mentioned in Section 2 , the predicted positron fraction well agrees with the PAMELA result if the lifetime of the gravitino is given by Eq. (2.2). In addition, in such a case, when $m_{3 / 2}$ is large enough, the electron and positron

\footnotetext{
${ }^{\# 7}$ It is often the case that, when the decay of dark matter produces hadronic objects, like $q \bar{q}$ pair, the cosmic-ray anti-proton flux becomes too large to be consistent with observations. However, the anti-proton flux is sensitive to the model of cosmic-ray propagation [25]. In addition, the ratio of the number of the $q \bar{q}$ pair to that of the positron is $\sim 6$ in the present scenario, which is about 4 times larger than the ratio for the scenario proposed in [14] where the heavy gauge boson for an exotic $U(1)$ gauge symmetry, which has a small kinetic mixing with the $U(1)_{B-L}$ gauge boson, is dark matter. The cosmic-ray anti-proton flux in such a scenario is order of magnitude smaller than the observed anti-proton flux. Thus, we expect that the constraint from the anti-proton flux does not exclude the present scenario. More detail of this subject will be discussed elsewhere [54].
} 
fluxes for the energy range of $100 \mathrm{GeV} \lesssim E \leq \frac{1}{2} m_{\mathrm{DM}}$ are approximately given by

$$
E^{2} \Phi_{e^{ \pm}} \simeq 3.0 \times 10^{-5} \mathrm{GeV} / \mathrm{cm}^{2} \sec \operatorname{str} \times\left(\frac{\tau_{3 / 2}}{8.5 \times 10^{25} \mathrm{sec}}\right)^{-1}\left(\frac{m_{3 / 2}}{1 \mathrm{TeV}}\right)^{-1},
$$

where $\tau_{3 / 2}$ is the lifetime of the gravitino. Then, as in the previous case, there exists a bump in the $\left(e^{-}+e^{+}\right)$flux at $E \sim 500 \mathrm{GeV}$ if we take $m_{3 / 2} \sim 1 \mathrm{TeV}$ (and the PAMELA best-fit value of the lifetime). This may be the origin of the excess of the $\left(e^{-}+e^{+}\right)$flux recently observed by the ATIC [27] and the PPB-BETS [28] experiments. (For detail, see [54].) On the contrary, if $l_{f}^{ \pm}=\mu^{ \pm}$and $\tau^{ \pm}, e^{ \pm}$from the decay of $l_{f}^{ \pm}$becomes non-monochromatic.

The electron and positron produced by the gravitino decay become the sources of the synchrotron radiation. In our numerical calculation, we include all the relevant interaction terms to calculate the partial decay rates; for detail, see [26] \#8 Then, for a precise calculation of the energy spectrum, we use the PYTHIA package [55] to calculate $d N_{e} / d E$.

First, we show the results for the case where $f=1$ (i.e., $l_{f}^{ \pm}=e^{ \pm}$), for which $J_{\nu}$ is maximized for the gravitino dark matter case. In Fig. 5, we plot the fluxes for $\nu=22 \mathrm{GHz}$ as functions of the gravitino mass, taking $\tau_{3 / 2}=5 \times 10^{26} \mathrm{sec}$. The angle $\theta$ is taken to be $5^{\circ}, 10^{\circ}, 15^{\circ}$, and $20^{\circ}$, and $B=1,3$, and $10 \mu \mathrm{G}$. One can see that the flux is maximized when the dark matter mass is slightly larger than $100 \mathrm{GeV}$. This is due to the fact that, when $m_{\mathrm{DM}} \sim 100 \mathrm{GeV}, \nu_{c}$ for $e^{ \pm}$from the decay of $X$ defined in Eq. (3.13) becomes comparable to $\nu \sim O(10 \mathrm{GHz})$.

The second case is that the primary lepton produced by the gravitino decay is in the second generation. The results are shown in Fig. 6. The synchrotron radiation flux becomes smaller in this case, compared to the case of $f=1$. This is because the $e^{ \pm}$produced by the decay of $\mu^{ \pm}$is less energetic than that directly produced by the process $\psi_{\mu} \rightarrow e^{ \pm} W^{\mp}$. We have also studied the case that $l_{f}^{ \pm}=\tau^{ \pm}$. As expected, the radiation flux becomes more suppressed in such a case.

For the gravitino dark matter case, the synchrotron radiation flux is again of the order of $\sim 1 \mathrm{kJy} / \mathrm{str}$ or smaller. As we have mentioned, we expect that the synchrotron radiation flux of this size is not excluded by the results of the presently available observations. The flux in the gravitino dark matter case is smaller than that for the dark matter which decays into $e^{+} e^{-}$pair (if the lifetime is fixed). This

\footnotetext{
\#8 There are typos in Eqs. (3.18) and (3.19) of [26]. The factors in front of the terms proportional to the function $G$ should be $\frac{2}{3}$, not $\frac{3}{2}$. We thank L. Covi for pointing out these typos.
} 


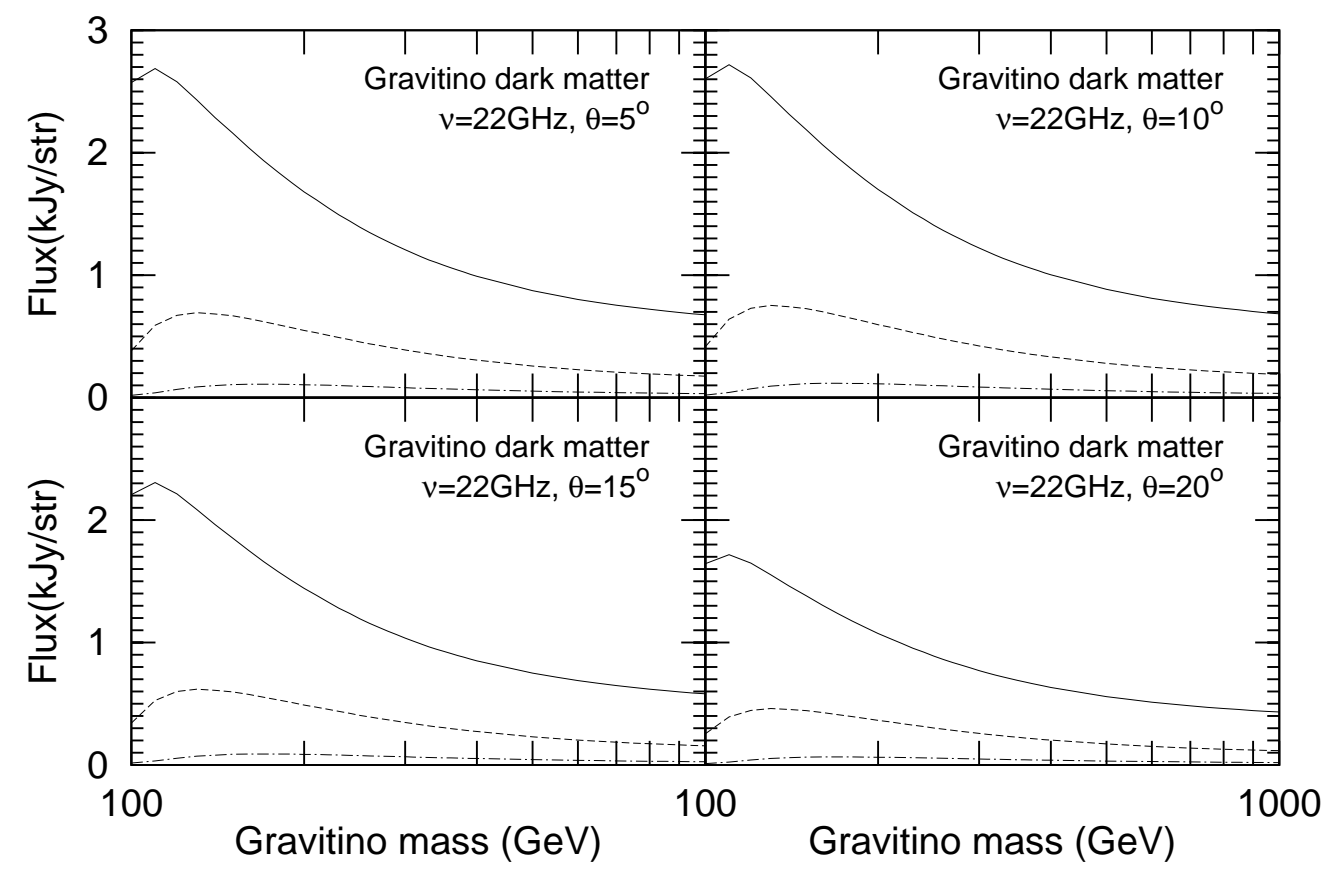

Figure 5: Synchrotron radiation fluxes at $\nu=22 \mathrm{GHz}$ as functions of gravitino mass for angle $\theta=5^{\circ}, 10^{\circ}, 15^{\circ}$, and $20^{\circ}$. The final-state lepton in the gravitino decay is in the first generation. Here, we take $\tau_{3 / 2}=5 \times 10^{26}$ sec, and show the cases of $B=1$, $3,10 \mu \mathrm{G}$ (from the bottom to the top) for each figure.

is due to the fact that the total amount of energy carried away by $e^{ \pm}$is smaller in the case of the gravitino dark matter.

\section{Summary}

In this paper, we have discussed the synchrotron radiation flux from the Galactic center in unstable dark matter scenario. Motivated by the recently reported PAMELA positron excess, we consider unstable dark matter whose decay produces energetic electron and positron; if the lifetime of dark matter is $O\left(10^{26-27} \mathrm{sec}\right)$, the observed positron flux can be well explained in such a scenario. Once the energetic $e^{ \pm}$is produced in the Galaxy, it becomes the source of the synchrotron radiation. As discussed in Section 3 , in our study, we have used a formalism with several improvements to calculate the synchrotron flux. Then, we have numerically calculated the flux for models which can well explain the PAMELA anomaly.

Assuming $\tau_{\mathrm{DM}} \sim O\left(10^{26} \mathrm{sec}\right)$ to explain the PAMELA anomaly, we found that 


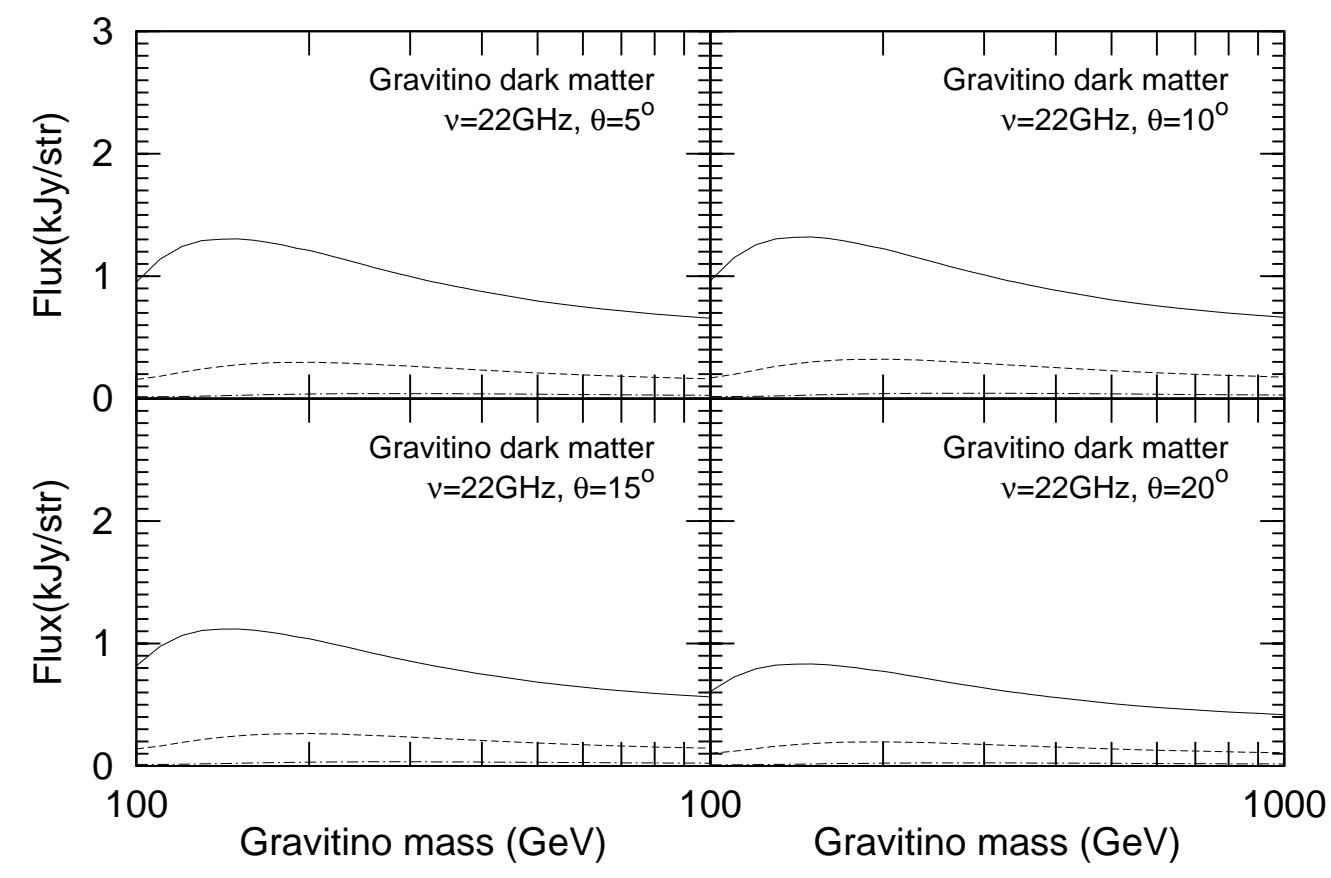

Figure 6: Same as Fig. 5, except for the case that the final-state lepton in the gravitino decay is in the second generation.

the synchrotron radiation flux from the dark matter decay is expected to be $J_{\nu} \sim$ $O(1 \mathrm{kJy} / \mathrm{str})$ (or smaller). As we have mentioned, the existence of the exotic radiation flux of this size is controversial. However, it should be also noted that the synchrotron radiation flux is inversely proportional to the lifetime of dark matter. In order to explain the PAMELA anomaly, the smaller value of $\tau_{\mathrm{DM}}$ is preferred as $m_{\mathrm{DM}}$ becomes lager. (See Eqs. (2.1) and (2.2).) Thus, if we consider too large $m_{\mathrm{DM}}$, it may become difficult to explain the PAMELA anomaly in the decaying dark matter scenario once we take into account the WMAP observation of the radiation from the central region of our Galaxy.

In any case, without better understandings of the sources of Galactic foreground emission, it is difficult to confirm or exclude the scenario of explaining the PAMELA anomaly in the decaying dark matter scenario (with $m_{\mathrm{DM}} \sim O(100 \mathrm{GeV})$ ). A more detailed study of the Galactic emission may provide a significant test of the unstable dark matter scenario. Once the existence of the WMAP Haze will be somehow confirmed, decaying dark matter may be a viable candidate of its origin.

Acknowledgements: This work was supported in part by Research Fellowships of the Japan Society for the Promotion of Science for Young Scientists (K.I.), and by the Grant-in-Aid for Scientific Research from the Ministry of Education, Science, Sports, 
and Culture of Japan, No. 19540255 (T.M.).

\section{References}

[1] O. Adriani et al., arXiv:0810.4995 [astro-ph].

[2] I. V. Moskalenko and A. W. Strong, Astrophys. J. 493, 694 (1998).

[3] E. A. Baltz and J. Edsjo, Phys. Rev. D 59, 023511 (1999).

[4] M. Cirelli, M. Kadastik, M. Raidal and A. Strumia, arXiv:0809.2409 [hep-ph].

[5] I. Cholis, D. P. Finkbeiner, L. Goodenough and N. Weiner, arXiv:0810.5344 [astro-ph].

[6] D. Feldman, Z. Liu and P. Nath, arXiv:0810.5762 [hep-ph].

[7] P. J. Fox and E. Poppitz, arXiv:0811.0399 [hep-ph].

[8] L. Bergstrom, T. Bringmann and J. Edsjo, arXiv:0808.3725 [astro-ph].

[9] V. Barger, W. Y. Keung, D. Marfatia and G. Shaughnessy, arXiv:0809.0162 [hep-ph].

[10] A. E. Nelson and C. Spitzer, arXiv:0810.5167 [hep-ph].

[11] R. Harnik and G. D. Kribs, arXiv:0810.5557 [hep-ph].

[12] P. f. Yin, Q. Yuan, J. Liu, J. Zhang, X. j. Bi and S. h. Zhu, arXiv:0811.0176 [hep-ph].

[13] K. Ishiwata, S. Matsumoto and T. Moroi, arXiv:0811.0250 [hep-ph].

[14] C. R. Chen, F. Takahashi and T. T. Yanagida, arXiv:0811.0477 [hep-ph].

[15] K. Hamaguchi, E. Nakamura, S. Shirai and T. T. Yanagida, arXiv:0811.0737 [hep-ph].

[16] E. Ponton and L. Randall, arXiv:0811.1029 [hep-ph].

[17] A. Ibarra and D. Tran, arXiv:0811.1555 [hep-ph].

[18] J. Hisano, S. Matsumoto and M. M. Nojiri, Phys. Rev. D 67, 075014 (2003).

[19] J. Hisano, S. Matsumoto and M. M. Nojiri, Phys. Rev. Lett. 92, 031303 (2004) arXiv:hep-ph/0307216.

[20] N. Arkani-Hamed, D. P. Finkbeiner, T. Slatyer and N. Weiner, arXiv:0810.0713 [hep-ph]. 
[21] D. Hooper, D. P. Finkbeiner and G. Dobler, Phys. Rev. D 76, 083012 (2007).

[22] D. Hooper, Phys. Rev. D 77, 123523 (2008).

[23] L. J. Hall, T. Moroi and H. Murayama, Phys. Lett. B 424, 305 (1998).

[24] T. Asaka, K. Ishiwata and T. Moroi, Phys. Rev. D 73, 051301 (2006).

[25] A. Ibarra and D. Tran, JCAP 0807, 002 (2008).

[26] K. Ishiwata, S. Matsumoto and T. Moroi, Phys. Rev. D 78, 063505 (2008).

[27] J. Chang et al., Nature 456, 362 (2008).

[28] S. Torii et al., arXiv:0809.0760 [astro-ph].

[29] T. Delahaye, R. Lineros, F. Donato, N. Fornengo and P. Salati, Phys. Rev. D 77,063527 (2008).

[30] G. B. Rybicki, A. P. Lightman, "Radiative Processes in Astrophysic," (John Wiley \& Sons, 1979).

[31] M. S. Longair, "High-energy astrophysics. Vol. 1: Particles, photons and their detection," (Cambridge, 1992); "High-energy astrophysics. Vol. 2: Stars, the galaxy and the interstellar medium," (Cambridge, 1994).

[32] A. W. Strong, I. V. Moskalenko and O. Reimer, Astrophys. J. 537, 763 (2000) [Erratum-ibid. 541, 1109 (2000)].

[33] P. Gondolo, Phys. Lett. B 494, 181 (2000).

[34] G. Bertone, G. Sigl and J. Silk, Mon. Not. Roy. Astron. Soc. 326, 799 (2001).

[35] R. Aloisio, P. Blasi and A. V. Olinto, JCAP 0405, 007 (2004).

[36] M. Regis and P. Ullio, Phys. Rev. D 78, 043505 (2008)

[37] L. Zhang and G. Sigl, JCAP 0809, 027 (2008).

[38] G. Dobler and D. P. Finkbeiner, Astrophys. J. 680, 1222 (2008).

[39] B. Gold et al. [WMAP Collaboration], arXiv:0803.0715 [astro-ph].

[40] D. P. Finkbeiner, M. Davis and D. J. Schlegel, Astrophys. J. 524, 867 (1999).

[41] D. P. Finkbeiner, Astrophys. J. 614, 186 (2004).

[42] D. P. Finkbeiner, Astrophys. J. Suppl. 146, 407 (2003).

[43] G. Hinshaw et al. [WMAP Collaboration], Astrophys. J. Suppl. 170, 288 (2007).

[44] F. Takayama and M. Yamaguchi, Phys. Lett. B 485, 388 (2000). 
[45] T. Moroi, H. Murayama and M. Yamaguchi, Phys. Lett. B 303, 289 (1993).

[46] J. L. Feng, A. Rajaraman and F. Takayama, Phys. Rev. Lett. 91, 011302 (2003).

[47] J. L. Feng, A. Rajaraman and F. Takayama, Phys. Rev. D 68, 063504 (2003).

[48] J. R. Ellis, K. A. Olive, Y. Santoso and V. C. Spanos, Phys. Lett. B 588, 7 (2004).

[49] L. Roszkowski, R. Ruiz de Austri and K. Y. Choi, JHEP 0508, 080 (2005).

[50] K. Ishiwata, S. Matsumoto and T. Moroi, Phys. Rev. D 77, 035004 (2008).

[51] M. Endo, F. Takahashi and T. T. Yanagida, Phys. Lett. B 658, 236 (2008).

[52] S. W. Barwick et al. [HEAT Collaboration], Astrophys. J. 482, L191 (1997).

[53] P. Sreekumar et al. [EGRET Collaboration], Astrophys. J. 494, 523 (1998).

[54] K. Ishiwata, S. Matsumoto and T. Moroi, in progress.

[55] T. Sjostrand, S. Mrenna and P. Skands, JHEP 0605, 026 (2006). 